\title{
Cariotipo del tití gris (Saguinus leucopus): similitudes con el cariotipo humano
}

\author{
Juan Hember Tabares', Carlos Humberto Fierro', \\ Paola Del Pilar Pulido', Humberto Ossa Reyes ${ }^{3}$
}

\begin{abstract}
1. Biólogo. Laboratorio de Genética y Biología Molecular Ltda.
2. Bióloga. Maestría en Ciencias Biológicas U. Nacional de Colombia

3. Biólogo Genetista. Laboratorio de Genética y Biología Molecular Ltda.
\end{abstract}

Correspondencia: hossa@cable.net.co, crazybiostica@gmail.com

Recibido: 25- 08-09 / 15-09-09

\begin{abstract}
Resumen
El tití gris (Saguinus leucopus) es un primate endémico de Colombia cuyo cariotipo se describe en el presente estudio a partir de una pareja de individuos ubicados en el Centro de Rehabilitación de Fauna Silvestre del Oriente de Caldas, Colombia. Las muestras de sangre fueron recolectadas de la vena femoral y anticoaguladas con heparina de sodio. Los cromosomas se obtuvieron por el método clásico de cultivo de linfocitos y bandeamiento Q y G Los individuos presentan 46 cromosomas $(2 n=46: 30 \mathrm{Bi}$, I $4 \mathrm{~A})$; cromosomas sexuales $X X$ en la hembra y $X Y$ en el macho (quimerismo 46,XX/46,XY en este último). Se propone un ideograma para el cariotipo del Tití Gris. Se observan amplias semejanzas en los cromosomas $X$ y 5 de $S$. leucopus con los cromosomas $X$ y 19 humanos, respectivamente. Otras similitudes parciales se evidenciaron entre los cromosomas I de ambas especies, 2 y 14 de $S$. leucopus con el 7 humano. La comparación del tamaño de regiones exónicas de dos genes de $\mathrm{S}$. leucopus y Homo sapiens no arrojó diferencia.

Palabras clave: cariotipo, cromosomas, primates, quimerismo, Saguinus leucopus.
\end{abstract}

\begin{abstract}
White-footed Tamarin (Saguinus leucopus) is a primate endemic to Colombia whose karyotype is described in this study from a pair of individuals located in the East of Caldas Wild Fauna Rehabilitation Center in Colombia. The blood samples were collected from the femoral vein and anti-coagulated with heparin sodium. The chromosomes obtained by the classic method of culture of lymphocytes as well as $Q$ and $G$ banding. The individuals display 46 chromosomes $(2 n=46: 30 \mathrm{Bi}, 14 \mathrm{~A})$; sexual chromosomes $X X$ in female and $X Y$ in the male (chimerism 46, $X X / 46, X Y$ in this last one). An ideogram for the White-footed Tamarin karyotype is proposed. Ample similarities in S. leucopus chromosomes Xy 5 are observed and with human chromosomes Xy 19, respectively. Other partial similarities were demonstrated between chromosomes I of both species, S. leucopus 2 and 14 with human 7. The comparison of the size of exonic regions of two genes of S. leucopus and Homo sapiens did not show any difference.
\end{abstract}

Key Words: chimerism, chromosomes, karyotype, Saguinus leucopus. 


\section{Introducción}

El tití gris (Saguinus leucopus) es un primate endémico de Colombia, Figura 1; se encuentra en el nordeste de Antioquia, sur de Bolívar, Caldas y norte del Tolima; su área de distribución es la más reducida de todas las especies de Saguinus. Su hábitat es el bosque seco tropical, húmedo tropical, muy húmedo tropical y muy húmedo premontano. Sus grupos están compuestos generalmente de 2 a 12 individuos y tienen una longevidad en vida salvaje de 18 años (1). Esta especie se ha visto afectada por la presión de caza, tráfico ilegal y reducción de su hábitat por actividad humana, siendo clasificada por la International Union for Conservation of Nature (IUCN) como especie en peligro de extinción (2). Leguizamón y colaboradores encontraron evidencia de un posible cuello de botella poblacional en 44 individuos de S. leucopus provenientes de diferentes centros de rehabilitación y de vida silvestre (3).

Los miembros de la familia Callithrichidae, a la cual pertenece $S$. leucopus, descienden de especies de mayor talla, que durante un período de varios millones de años han adquirido uñas tipo garra, perdiendo el pulgar

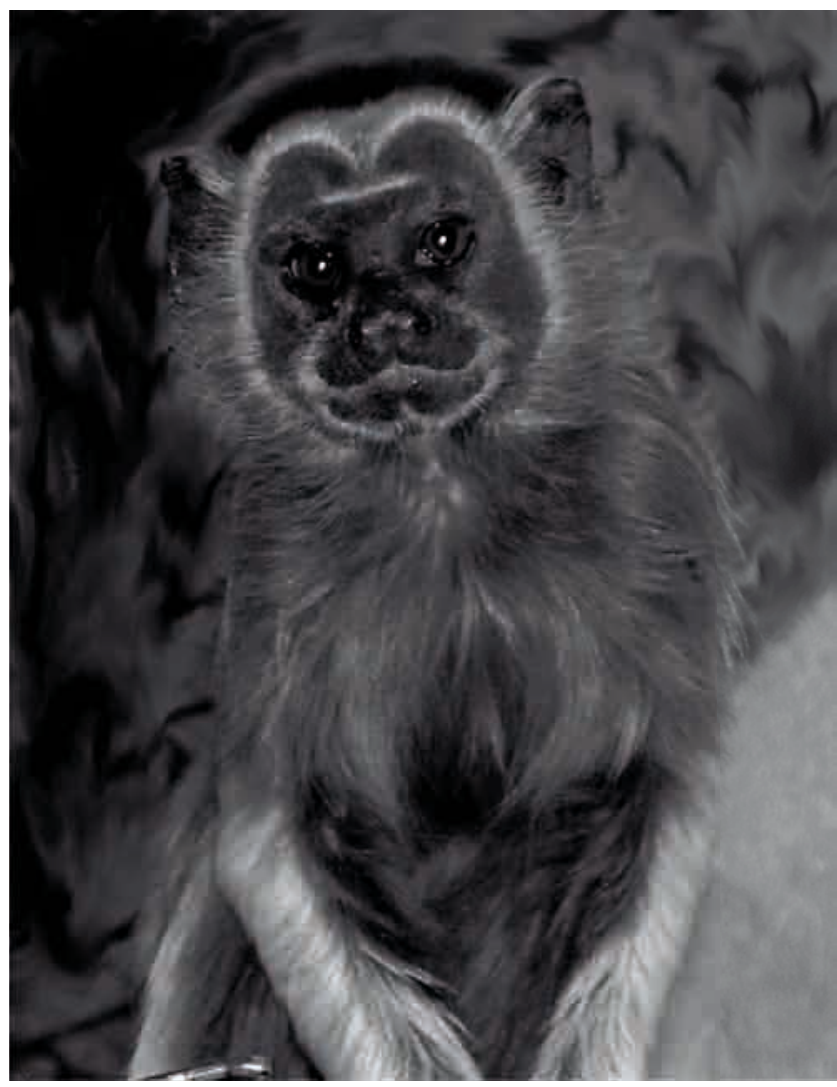

Figura 1. Individuo de la especie S. leucopus.

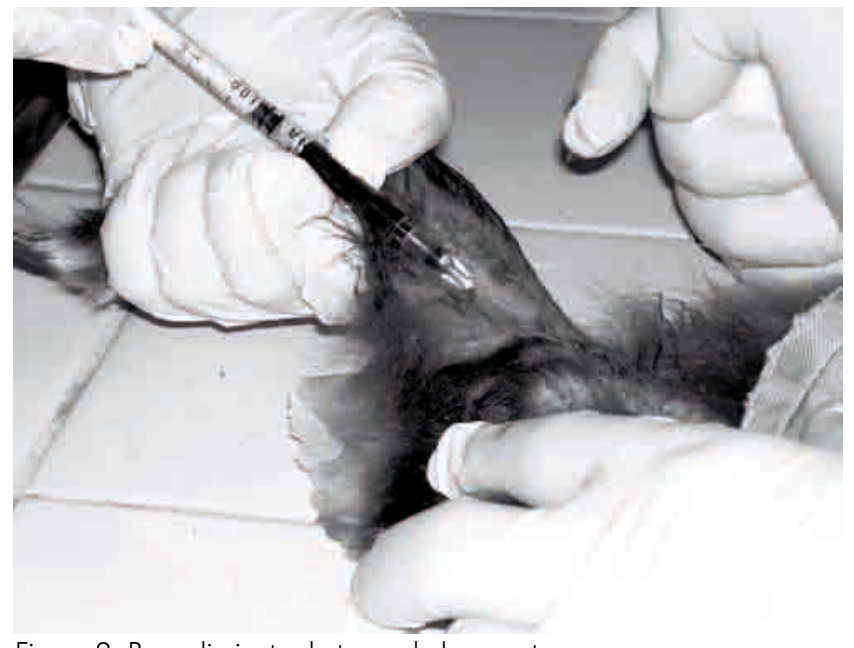

Figura 2. Procedimiento de toma de la muestra.

oponible en las manos y el tercer molar; además, tienen la característica de presentar partos múltiples, siendo el gemelar el más común (4). Los reportes moleculares de Schneider y su equipo (5) demostraron que Saguinus es el género base de los Callithrichidae; también los estudios citogenéticos han llevado a esta conclusión (6).

Nagamachi y Pieczarka presentaron el cariotipo de S. midas niger, estudiado por bandas G, Cy NOR (7). En este aspecto, se destaca el estudio de Mendes y de Souza, que establecieron los cariotipos de ocho especies brasileñas de Saguinus ( $S$. fuscicollis fuscicollis, S. fuscicollis weddelli, S. bicolor bicolor, $S$. bicolor martinsi, S. mystax mystax, S. imperator imperator, $S$. midas midas y $S$. midas niger) (8). El objetivo de este estudio fue analizar el cariotipo del tití gris (Saguinus leucopus) y compararlo con el cariotipo humano.

\section{Materiales y métodos}

Métodos citogenéticos. Los animales fueron sedados con ketamina y se tomaron muestras de sangre de la vena femoral, las cuales fueron anticoaguladas con heparina sódica, Figura 2. Los cromosomas se obtuvieron por el método clásico de cultivo de linfocitos (9), variando la cantidad de muestra empleada a solo un $50 \%$ de la que se utiliza en los cultivos para citogenética humana. Se emplearon las técnicas desarrolladas por Caspersson y colaboradores (10) y por Seabright (11) para obtener los patrones de bandeamiento Qy G, respectivamente, Figura 2. 


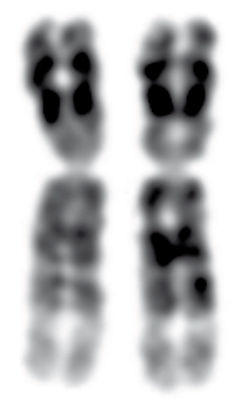

1

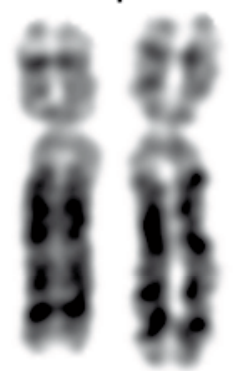

6

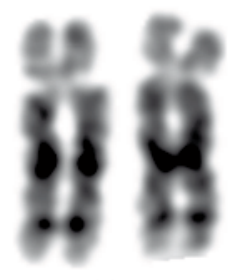

11

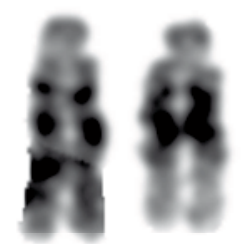

16

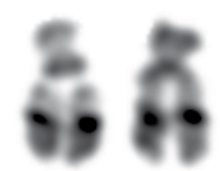

21

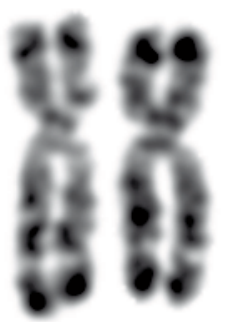

2

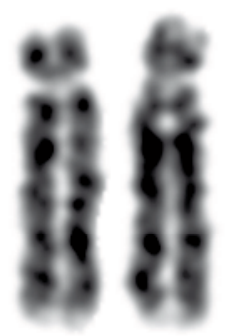

7

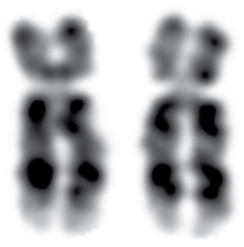

12

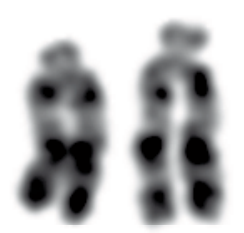

17

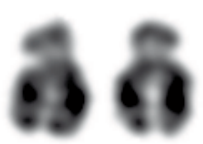

22

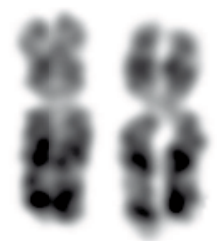

3

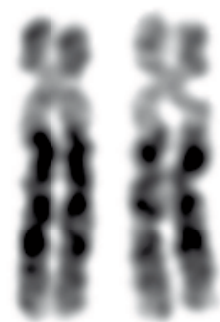

8

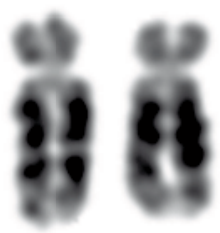

13

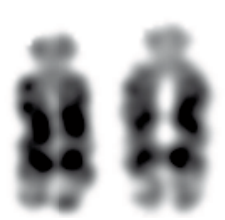

18

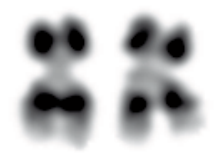

4

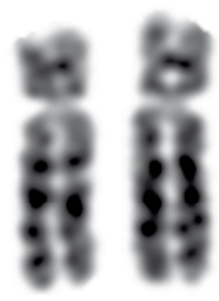

9

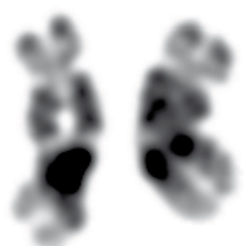

14

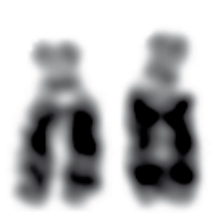

19

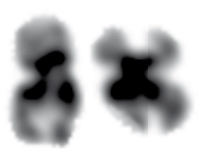

5

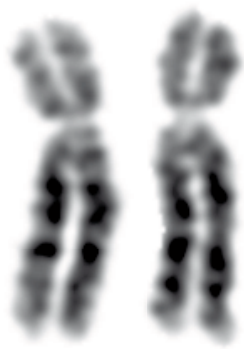

10

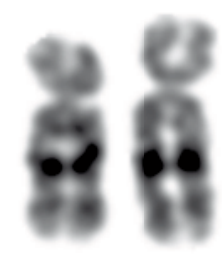

15

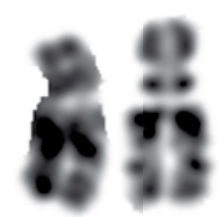

20

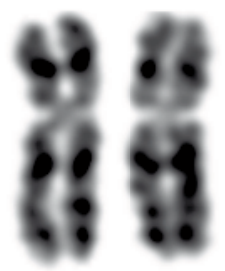

$X$

Figura 3. Cariotipo 46, XX de una hembra S. leucopus.

Las imágenes de bandas $\mathrm{Q}$ fueron tomadas en microscopio de fluorescencia (Olympus BX50) bajo aumento de $1000 \mathrm{X}$ y procesadas en el software KaryoView (Elja Inc.); se invirtieron los tonos de las metafases para facilitar el análisis: las bandas oscuras corresponden a las zonas más fluorescentes. Las imágenes de bandas $\mathrm{G}$ se tomaron en microscopio de luz blanca (Olympus BX50) a un aumento de 1000X y se procesaron sin ninguna novedad en el software KaryoView (Elja Inc.). En total se capturaron y analizaron 50 metafases de la hembra y 50 del macho. A partir de las metafases obtenidas se elaboró un ideograma para el cariotipo de S. leucopus. El orden de los cromosomas en el cariotipo se asignó teniendo como referencia el trabajo de las investigadoras brasileñas Mendes y de Souza (8). Se comparó el 
cariotipo del tití gris con el humano. Los cariotipos humanos utilizados en la comparación pertenecen a individuos normales.

Métodos moleculares. El ADN de S. leucopus al igual que el de $H$. sapiens fue aislado mediante el kit Wizard (Promega) y posteriormente amplificado para una región de $345 \mathrm{pb}$ del gen de la protrombina humana (Factor II) y una región de 203 pb del gen Metilentetrahidrofolato
Reductasa (MTHFR) (12). Posteriormente, se procedió a realizar digestión enzimática con Mnl I y Hinf I, respectivamente, para determinar la presencia de las mutaciones G20210A para el Factor II y C677T para el MTHFR, las cuales se asocian en humanos a enfermedad vascular y ateroesclerosis (13). La electroforesis de los productos de digestión se realizó en gel de agarosa al 2\% y su visualización fue y con bromuro de etidio. El ADN

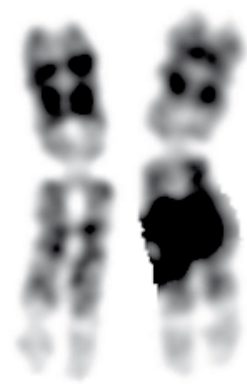

1

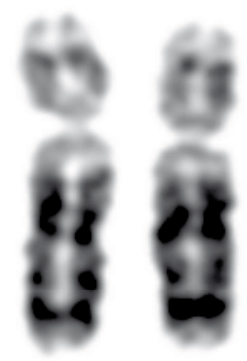

6

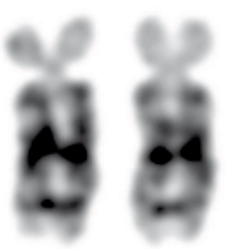

11

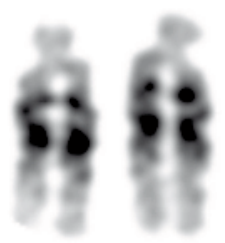

16

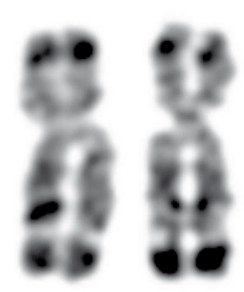

2

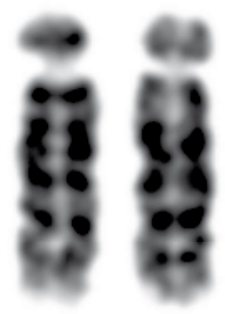

7

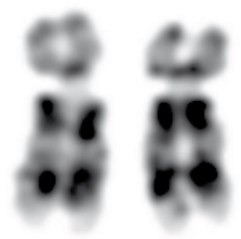

12

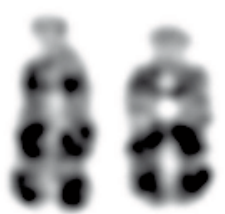

17

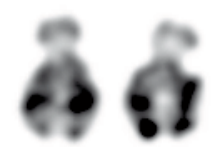

22

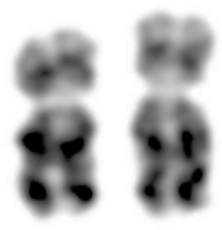

3

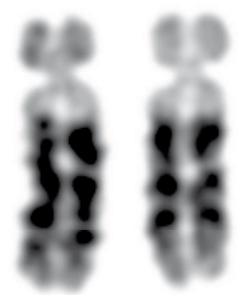

8

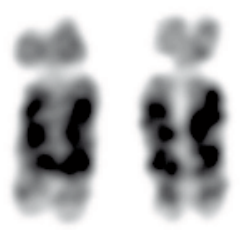

13

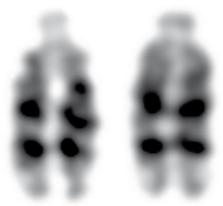

18

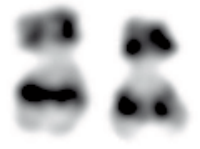

4

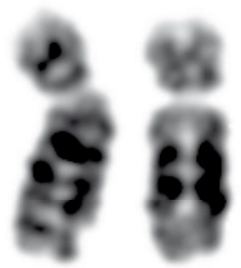

9

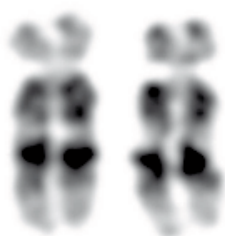

14

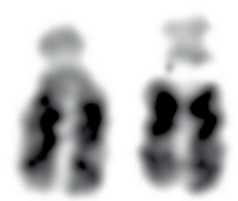

10

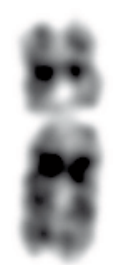

X

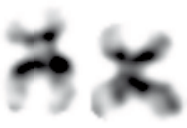

5

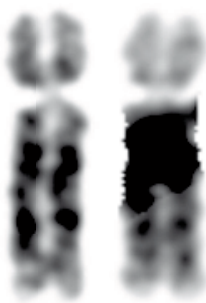

10

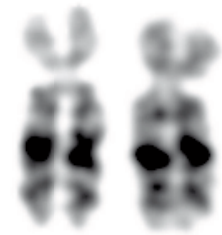

15

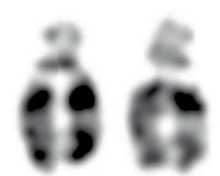

20

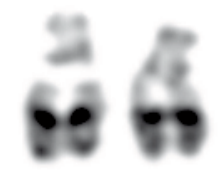

21

Figura 4. Cariotipo 46,XY de un macho S. leucopus. Las zonas altamente fluorescentes de lq y 10q son el resultado de una sobreposición de estos cromosomas en la metafase, no representan bandas de los cromosomas. 
humano pertenece a controles sanos, libres de las mutaciones.

\section{Resultados}

Se encontraron 46 cromosomas $(2 n=46)$ en el cariotipo de la hembra y el macho examinados, de los cuales 30 poseen dos brazos (30Bi) y 14 son acrocéntricos (14A), Figuras 3 y 4 . Los cromosomas sexuales de la hembra son XX; el macho presenta cromosomas sexuales $\mathrm{XY}$, pero se observa quimerismo 46,XX/46XY, un hecho que se ha descrito en otras especies de Saguinus (8). El ideograma del cariotipo de $S$. leucopus presenta una resolución promedio de 300-400 bandas, Figura 5.

Se observó similitud completa entre los cromosomas $\mathrm{X}$ de ambas especies, Figura 6, A,B,C; asimismo, entre el cromosoma 5 de S. leucopus y el 19 del ser humano, Figura 6, D- F. El brazo corto del cromosoma 7 humano presenta similitud con $2 \mathrm{p}$ de $S$. leucopus; $7 \mathrm{q}$ de $H$. sapiens tiene correspondencia con 12q del tamarino, Figura 7, A-E. Los satélites de los cromosomas acrocéntricos del tití gris presentan una configuración distinta a la de los cromosomas acrocéntricos humanos, Figura 7, F y G.
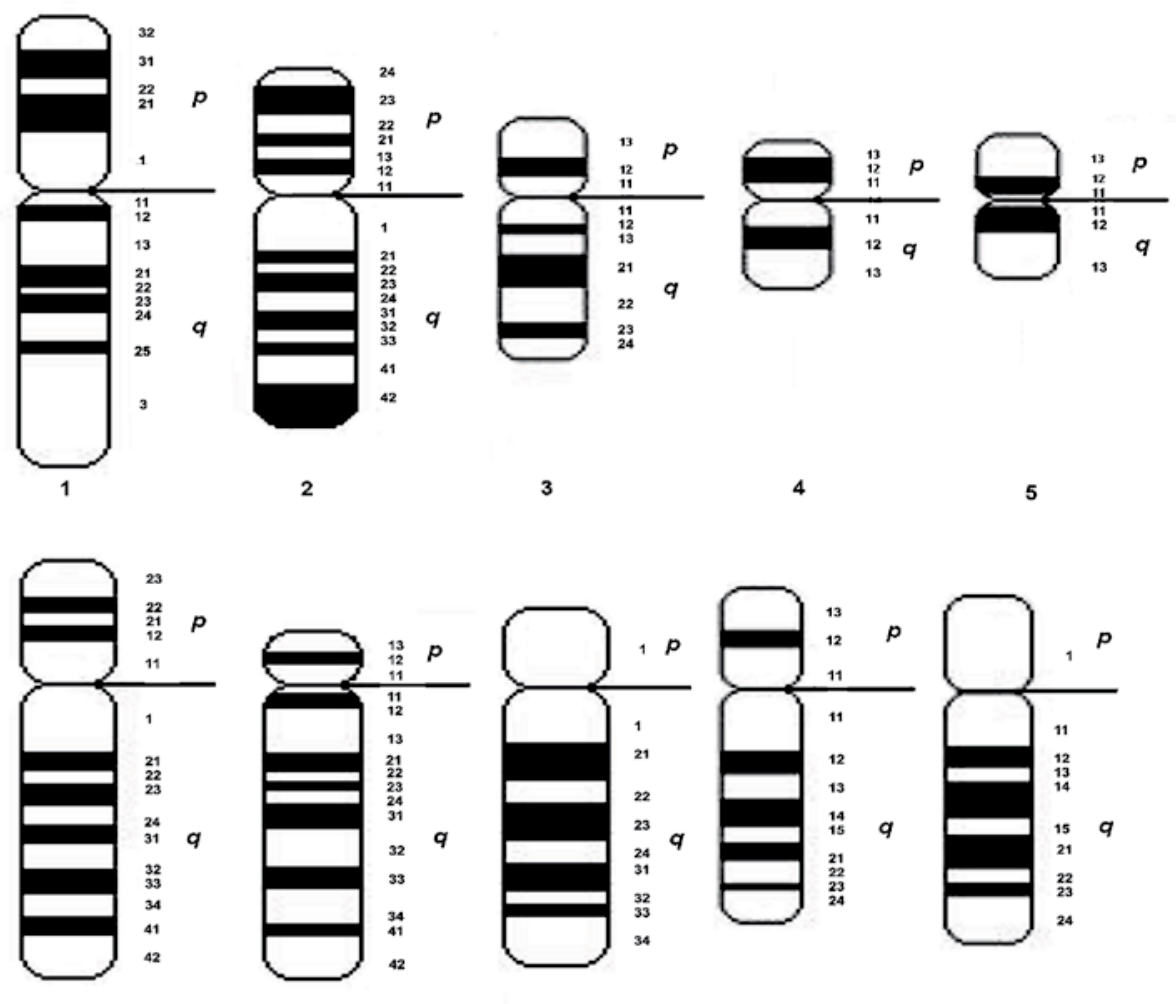

6
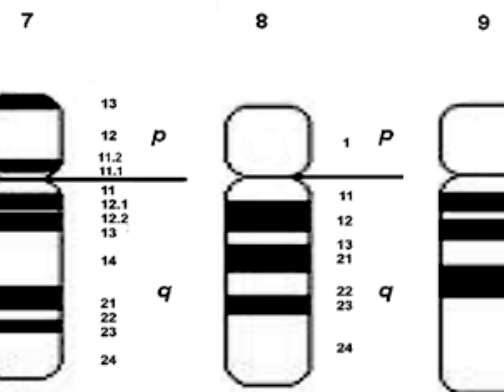

10
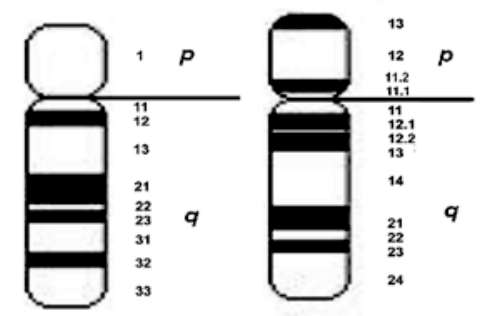

12
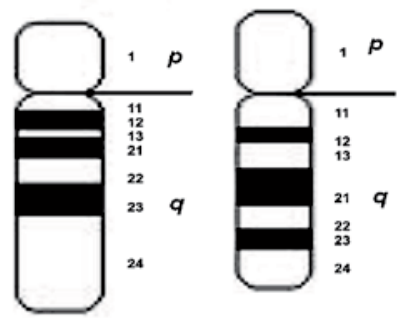

11

13

14

15

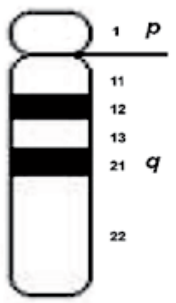

16

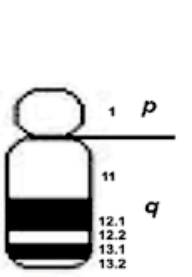

21

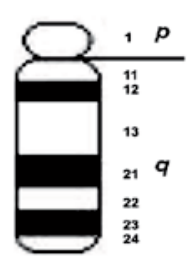

17

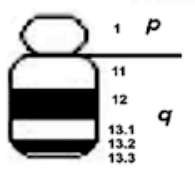

22

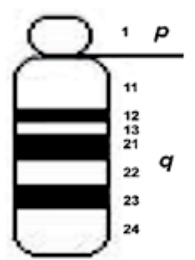

18

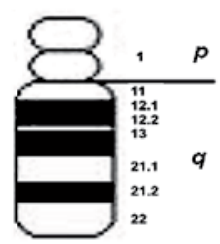

19

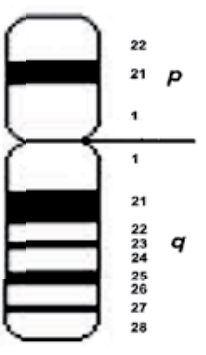

$\mathrm{x}$

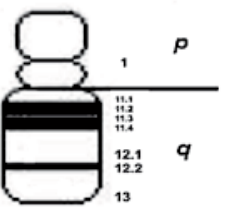

20

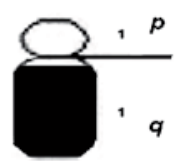

Figura 5. Ideograma del cariotipo de S. leucopus, resolución 300-400 bandas. 
A

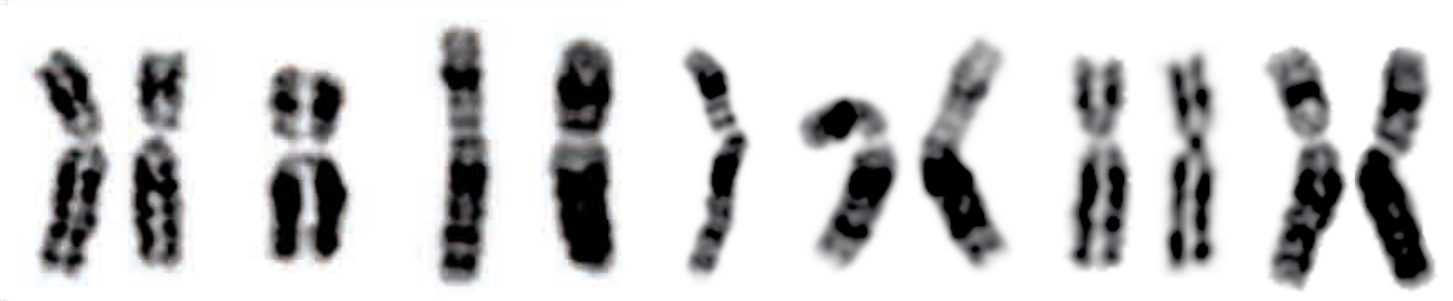

B

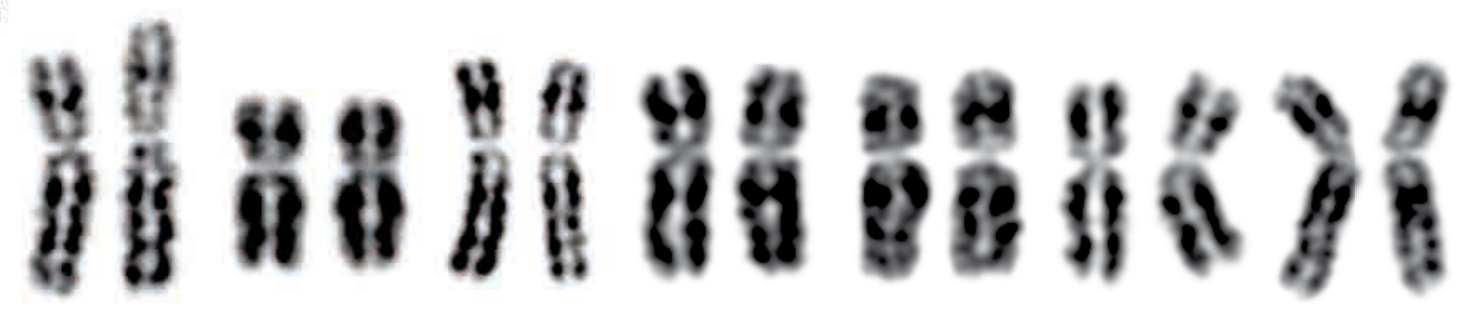

C

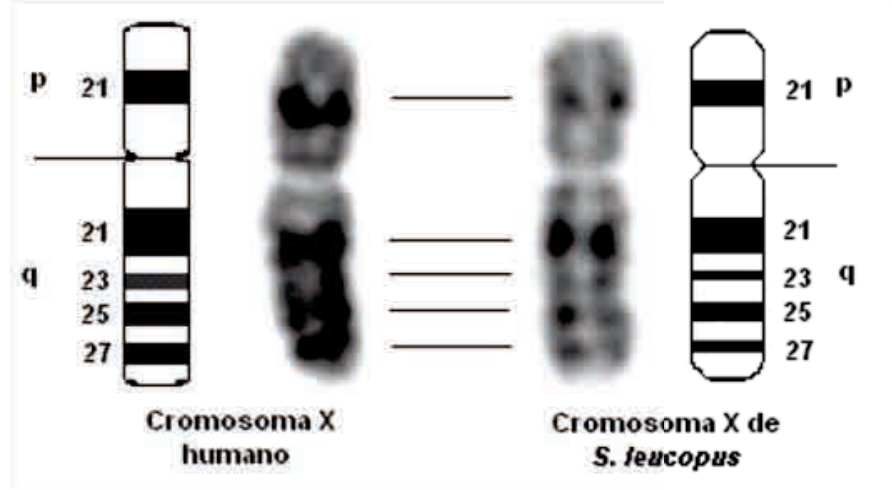

D

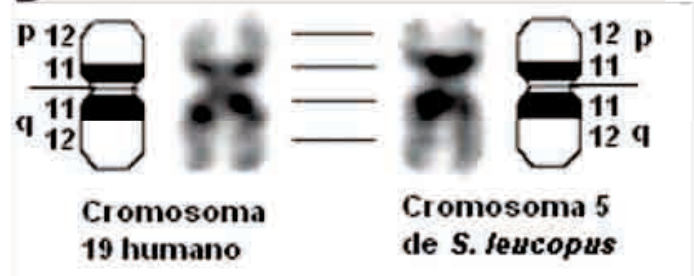

\section{E

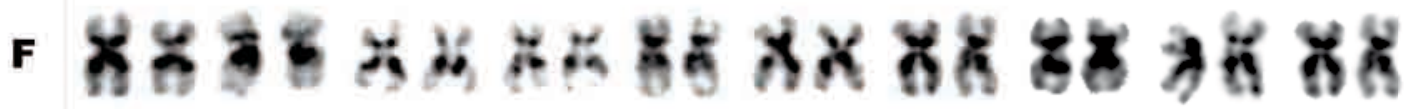

Figura 6. A. Cromosomas X de diferentes personas. B. Cromosomas X de S. leucopus. C. Comparación del bandeamiento entre cromosomas $X$ de las dos especies. D. Comparación del bandeamiento entre cromosomas 19 de H. sapiens y 5 de S. leucopus. E. Cromosomas 19 de diferentes personas. F. Cromosomas 5 de S. leucopus.

El cromosoma 1 en ambas especies presenta gran similitud. Si el cromosoma 1 de $S$. leucopus es rotado $180^{\circ}$, se observa que todo el brazo q se asemeja a $1 \mathrm{p}$ humano, con una posible inversión que involucra la región central del brazo, Figura 8, A-C. No obstante, la gran región heterocromática de 1q12 humano parece estar ausente en $1 \mathrm{p}$ de $S$. leucopus. No se puede afirmar con certeza la presencia de la banda oscura 1q31 distintiva del cariotipo humano en 1 p de $S$. leucopus siendo difícil la determinación citogenética del origen del brazo corto del cromosoma 1 del tití gris. 
A
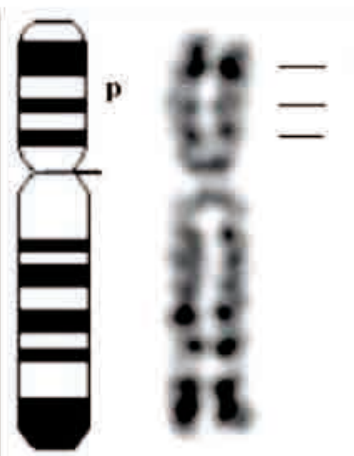

Cromosoma 2 de S. leucopus.

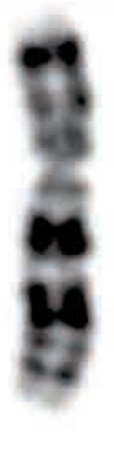

Cromosoma 7 humano

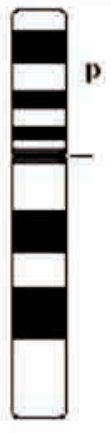

B
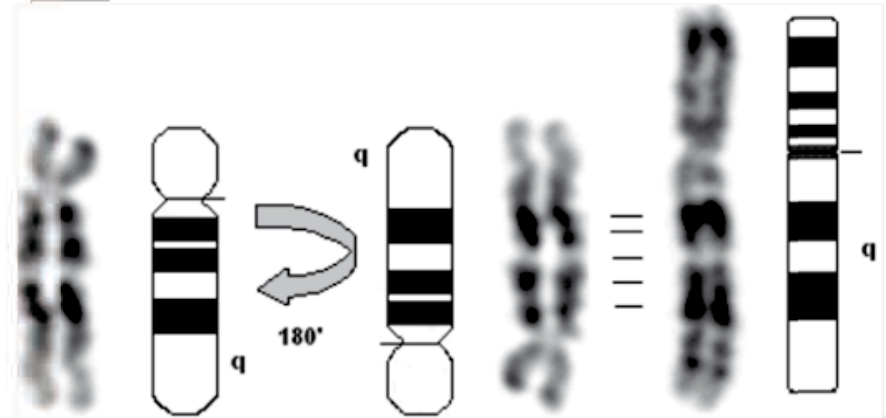

Cromosoma 14 de S. leucopus.
Cromosoma 7 humano.
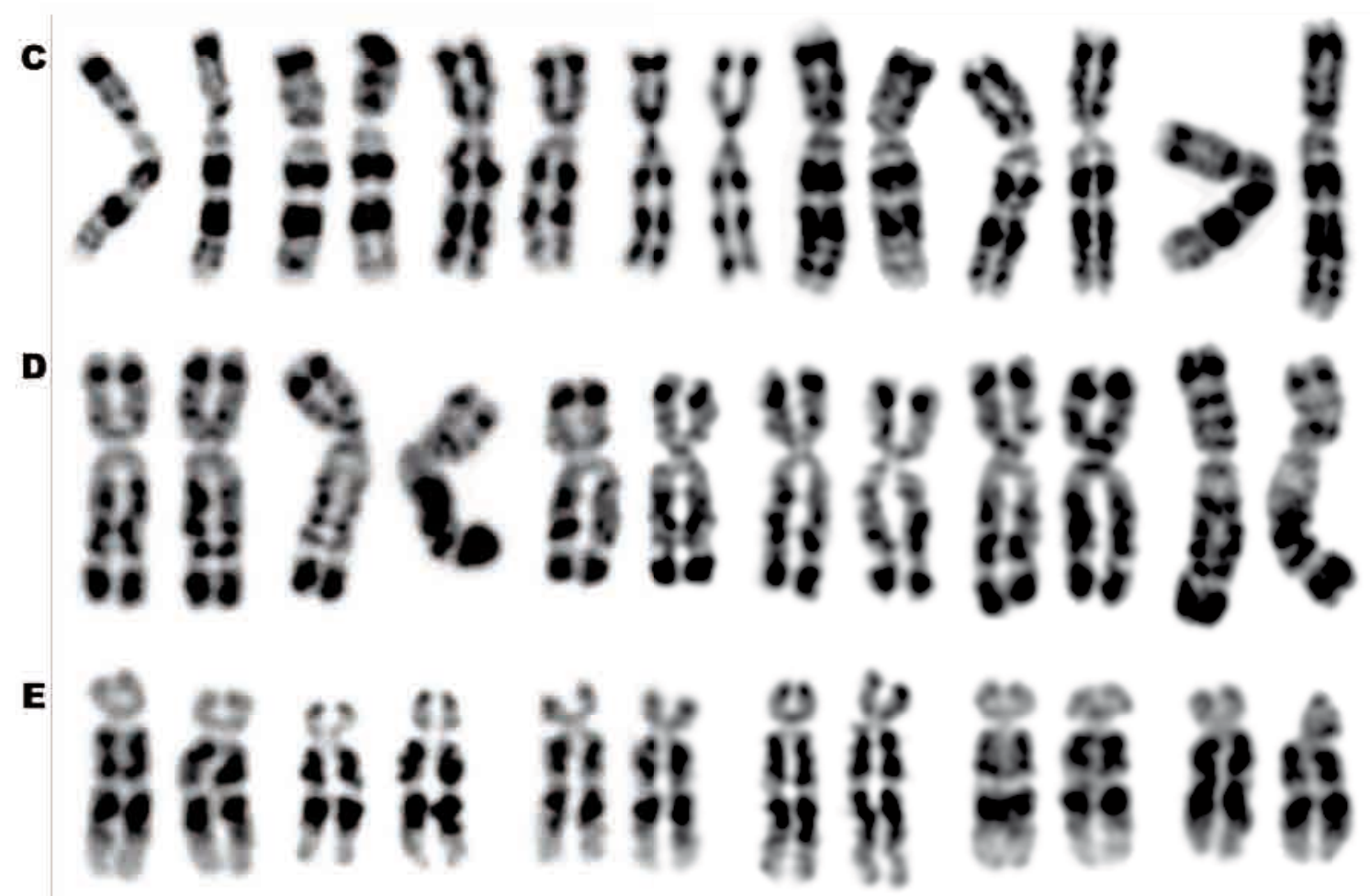

$\mathbf{F}$

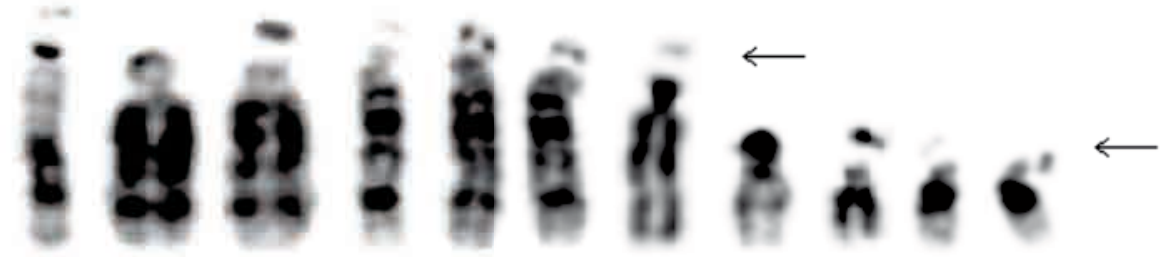

$\mathbf{G}$

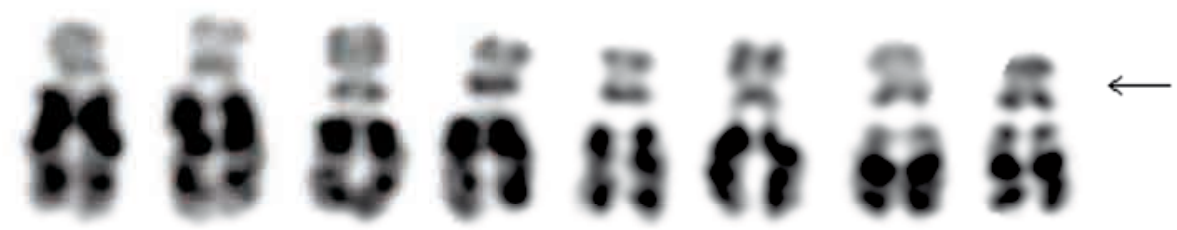

Figura 7. A. Comparación del bandeamiento entre el brazo corto del cromosoma 2 de S. leucopus y el brazo corto del cromosoma 7 de H. sapiens. B. Comparación del bandeamiento entre el brazo largo del cromosoma 14 de S. leucopus y el brazo largo del cromosoma 7 de H. sapiens. C. Cromosomas 7 de diferentes personas. D. Cromosomas 2 de S. leucopus. E. Cromosomas 14 de S. leucopus. F. Cromosomas acrocéntricos humanos. G. Cromosomas acrocéntricos de $\mathrm{S}$. leucopus. Obsérvese la particular configuración doble de satélites en $\mathrm{S}$. leucopus, que no se observa en seres humanos. 
A

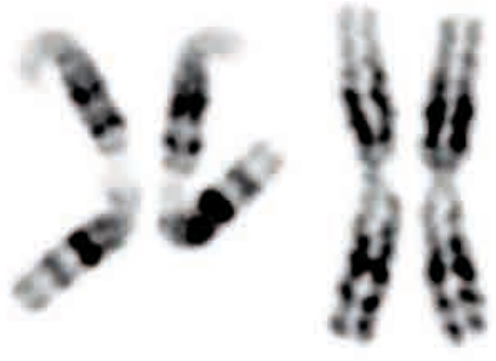

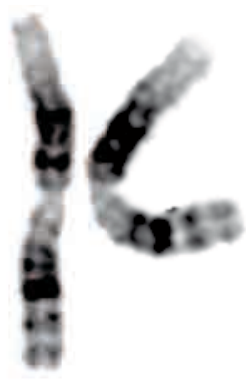
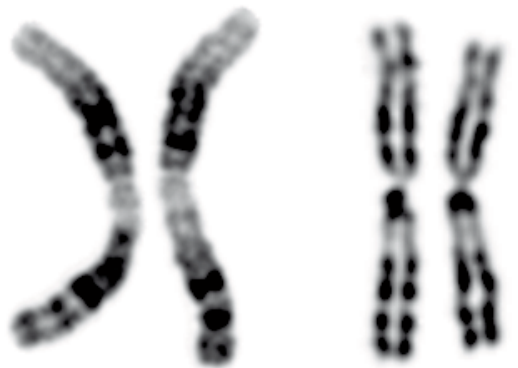

B

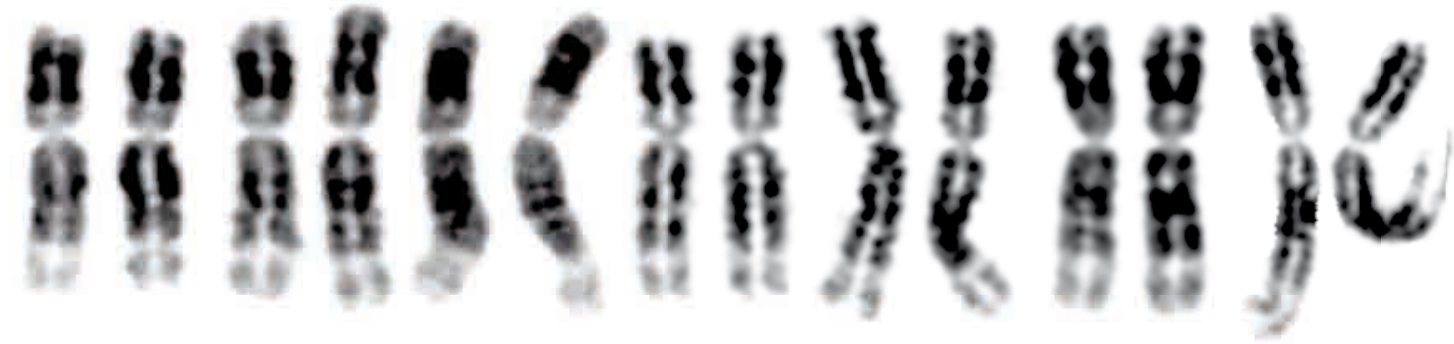

C

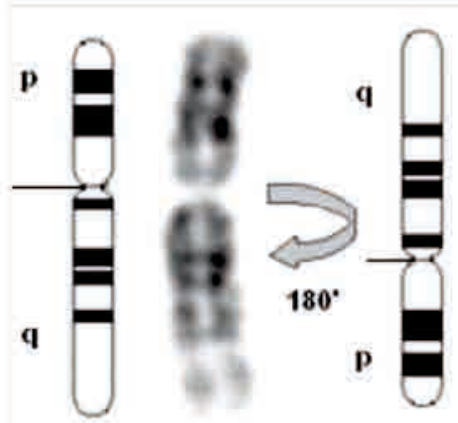

Cromosoma 1 de S. Seucopus

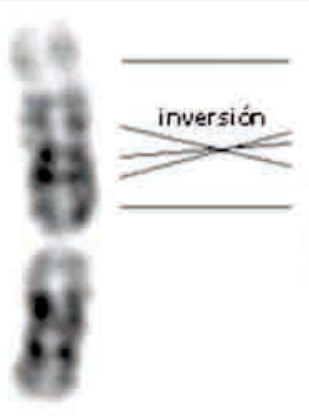

D
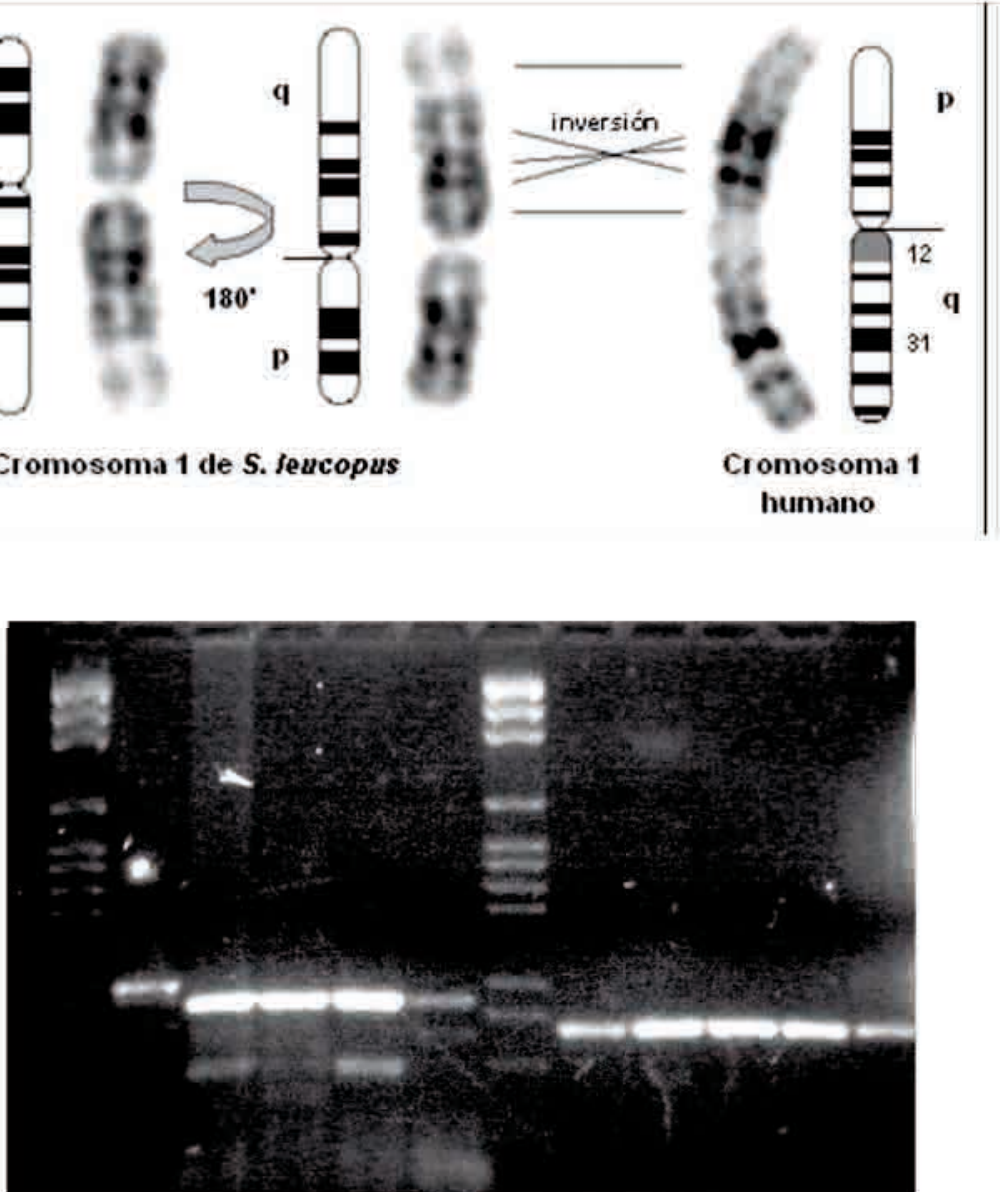

Figura 8. A. Cromosomas 1 de diferentes personas. B. Cromosomas 1 de S. leucopus. C. Comparación del bandeamiento entre cromosomas 1 de las dos especies. D. Gel del estudio molecular, carriles 1-6 Factor II, carriles 7-12 MTHFR. Carril 1: escalera Pgem; carril 2: S. leucopus hembra (sin Digestión); carril 3: S. leucopus hembra; carril 4: S. leucopus macho; carril 5: H. sapiens Nำ ; carril 6: H. sapiens N⒉ Carril 7: escalera Pgem; carril 8: S. leucopus macho (sin Digestión) 9. S. leucopus hembra; carril10: S. leucopus macho; carril 11: H. sapiens $\mathrm{N}^{\circ} 1$; carril 12: H. sapiens $\mathrm{N}^{\circ} 2$. 


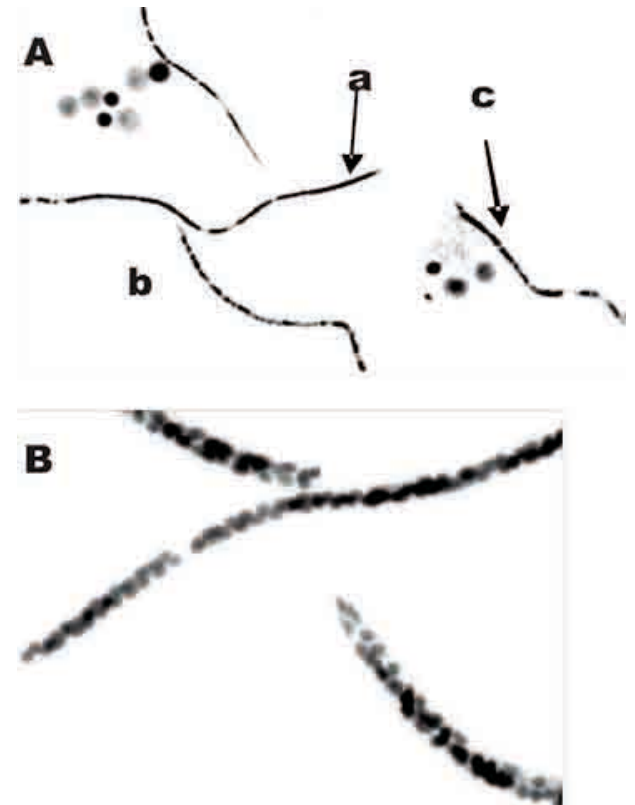

Figura 9. A. a. Microfilaria, b. Núcleos, c. Metafase. Aumento 400X. B. Microfilaria, aumento 1000X

Los procedimientos moleculares dieron lugar a la banda de amplificación de $345 \mathrm{pb}$ para el Factor II y 203 pb para el MTHFR tanto en S. leucopus como en $H$. sapiens, siendo evidente la similitud (al menos en el tamaño de la región amplificada de los dos genes) entre las dos especies. Los individuos estudiados tanto de $S$. leucopus como de $H$. sapiens no presentaron las mutaciones G20210A para el FII y C677T para el MTHFR, Figura 8D.

Se observó que la pareja de tamarinos presentaban infección por un parásito cuya morfología encaja con microfilaria, una fase larval de nemátodos parásitos que se encuentran en la corriente sanguínea. Fox y colaboradores (14) durante un estudio de la bioquímica hematológica de $S$. leucopus cautivos, encontraron que 22 de sus 29 individuos tenían microfilaremia, condición que se vio en los animales de la presente investigación, Figura 9.

\section{Discusión}

Desde el último ancestro común de las dos especies, hace 23 millones de ańos (5), los cromosomas de los antepasados de S. leucopus y H. sapiens pudieron sufrir rearreglos tales como fisiones, fusiones, inversiones, translocaciones, entre otros, las cuales hacen difícil determinar a priori la localización de los genes homólogos del ser humano en la otra especie. Sin embargo, al realizar una inspección citogenética cuidadosa, se pueden encontrar cromosomas o secciones cromosómicas comparables entre las dos especies, las cuales no parecen haber alterado drásticamente su estructura durante el proceso evolutivo. Esto sucede con el cromosoma X y el cromosoma 5 del tití gris que tienen su contraparte en el humano. El cromosoma 7 humano podría encontrarse en dos cromosomas distintos de $S$. leucopus.

Según Murphy y su equipo el cromosoma 1 humano parece haberse conservado muy bien respecto al cromosoma ancestral del que se originó en el antepasado de todos los mamíferos placentarios (15). El brazo q del cromosoma 1 alberga dos grupos importantes de hotspots para rupturas, lo que hace difícil encontrarlo intacto en muchas especies; creemos que $S$. leucopus es una de ellas.

Por otra parte, para explicar el cariotipo quimérico del tití gris macho estudiado, nos remitimos a lo observado en seres humanos, donde el quimerismo congénito se da gracias a la persistencia de transfusión de células madre hematopoyéticas durante el desarrollo fetal, que puede permanente o durar por muchos ańos $(16,17)$. En consecuencia, el quimerismo observado en el tamarino macho se puede explicar por una transfusión materno fetal o feto fetal en el útero materno, siendo probable en esta especie de primates donde los embarazos múltiples son frecuentes. En el trabajo de Mendes y de Souza (8) se reportó quimerismo 46,XX/46, XY en todas las especies de Saguinus analizadas.

Para nuestro conocimiento, esta es la primera descripción del cariotipo de $S$. leucopus, en la que adicionalmente se ha hecho un análisis objetivo de los posibles cromosomas y bandas que no se han visto modificadas durante la evolución en ambas especies. Esto arroja resultados interesantes, que nos conducen a afirmar que si se emplean sondas fluorescentes para genes humanos de los cromosomas 1p, 7, 19, y X, estamos en capacidad de predecir el sitio blanco al cual hibridarán en el cariotipo de $S$. leucopus, y por lo tanto, la ubicación de los genes en el genoma del tamarino.

Los resultados de este estudio ayudarán a la potencial identificación del origen geográfico de los $S$. leucopus que han llegado a los centros de rescate para su reincorporación al hábitat natural de los animales que aprueben los procesos de rehabilitación médico biológica. Para esto 
es necesario contrastar los resultados obtenidos en esta investigación con los análisis genético poblacionales propuestos por otros investigadores (3), así como integrar estos resultados en dichos análisis. La identificación de grupos poblacionales y su distribución facilitará la asignación de territorios nacionales para generar áreas de conservación específicas para el tití gris.

\section{Agradecimientos}

Centro de Rehabilitación de Fauna Silvestre del Oriente de Caldas. Corporación Autónoma Regional de Caldas (CORPOCALDAS). Asociación de Veterinarios de Vida Silvestre (V.V.S).

\section{Referencias}

1. Poveda K. Uso de hábitat de dos grupos de tití de pies blancos, Saguinus leucopus, en Mariquita, Colombia. Trabajo de grado. Universidad Nacional de Colombia. Bogotá. 2000.

2. IUCN Lista roja. http://www.iucnredlist.org/details/19819. 2008.

3. Leguizamón N, Ruiz-García M, Castillo MI. Aplicaciones de los análisis genético poblacionales a partir de genotipos multilocus y metodologías basadas en modelos bayesianos para la conservación del primate Saguinus leucopus. Revista: Conservación ex situ. Investigación para el manejo en cautiverio y conservación de la fauna silvestre. Editorial Leguizamón. Departamento Técnico Administrativo de la Alcaldía Mayor de Bogotá. 2006.

4. Carroll B. Guías para el estudio de calitrícidos. Bristol Zoo Gardens, 2002.

5. Scheneider H. $\otimes$ e Current Status of the New World Monkey Phylogeny. An Acad Bras Ciênc 2000;72:165-172.

6. Nagamachi CY, Pieczarka JC, Muniz JA, Barros RM, Mattevi MS. Proposed chromosomal phylogeny for the South American primates of the Callitrichidae family (Platyrrhini). Am J Primatol. 1999; 49:133-152.
7. Nagamachi, C.Y. and Pieczarka, J.C. Chromosome studies of Saguinus midas niger (Callithrichidae, Primates) from Tucuruí, Pará, Brazil: Comparison with the karyotype of Callithrix jacchus. Am J Primatol. 1988; 14:277-284.

8. Mendes SM, de Souza RM. Cytogenetic study of the genus Saguinus (Callithrichidae, Primates). Braz J Genet. 1997;20:1-5.

9. Moorhead PS, Norwell PC, Melman WJ, Battips DM and Hungerford DA. Chromosome preparation of leucocytes cultured from human peripheral blood. Exp Cell Res. 1960;20:613-615.

10. Caspersson T, Zech 1, Johansson, C, Modest EJ. Identification of human chromosomes by DNA-binding fluorescent agents. Chromosoma. 1970;30:215-227.

11. Seabright, M. Rapid banding techniques for human chromosomes. Lancet 1971;2: 971-972.

12. Poort SR, Rosendaal FR, Reitsma PH, Bertina RM. A common Genetic Variation in the 3'Unstranslated region for the Prothrombin gene is associated with elevated plasma Prothrombin levels and an increase in venous thrombosis. Blood 1996;88:3698-3703.

13. Koksal V, Baris L, Etlik O. Primer-engineered multiplex PCRRFLP for detection of MTHFR C677T, Prothrombin G2021OA and Factor V Leiden mutations. Exp Mol Pathol 2007;83:1-3.

14. Fox M, Brieva C, Moreno C, MacWilliams P, Thomas C. Hematologic and serum biochemistry reference values in wildcaught white-footed tamarins (Saguinus leucopus) housed in captivity. J Zoo Wildl Med. 2008;39:548-557.

15. Murphy WJ, Frönicke L, O’Brien SJ and Stanyon R. \ e origin of human chromosome 1 and its homologs in placental mammals. Genome Res. 2003;13:1880-1888.

16. Evans PC, Lambert N, Maloney S, Furst DE, Moore JM, Nelson JL. Long-term fetal microchimerism in peripheral blood mononuclear cell subsets in healthy women and women with scleroderma. Blood. 1999;93:2033-2037.

17. Johnson KL, Nelson JL, Furst DE, McSweeney PA, Roberts DJ, Zhen DK, Bianchi DW. Fetal cell microchimerism in tissue from multiple sites in women with systemic sclerosis. Arthritis Rheum. 2001;44:1848-1854. 